\title{
Magnetopause energy transfer dependence on the interplanetary magnetic field and the Earth's magnetic dipole axis orientation
}

\author{
M. Palmroth ${ }^{1}$, R. C. Fear ${ }^{2}$, and I. Honkonen ${ }^{1,3}$ \\ ${ }^{1}$ Finnish Meteorological Institute, Helsinki, Finland \\ ${ }^{2}$ Department of Physics \& Astronomy, University of Leicester, Leicester, UK \\ ${ }^{3}$ University of Helsinki, Department of Physics, Helsinki, Finland \\ Correspondence to: M. Palmroth (minna.palmroth@fmi.fi)
}

Received: 15 November 2011 - Revised: 24 February 2012 - Accepted: 27 February 2012 - Published: 7 March 2012

\begin{abstract}
We examine the spatial variation of magnetospheric energy transfer using a global magnetohydrodynamic (MHD) simulation (GUMICS-4) and a large data set of flux transfer events (FTEs) observed by the Cluster spacecraft. Our main purpose is to investigate whether it is possible to validate previous results on the spatial energy transfer variation from the GUMICS-4 simulation using the statistical occurrence of FTEs, which are manifestations of magnetospheric energy transfer. Previous simulation results have suggested that the energy transfer pattern at the magnetopause rotates according to the interplanetary magnetic field (IMF) orientation, and here we investigate whether a similar rotation is seen in the locations at which FTE signatures are observed. We find that there is qualitative agreement between the simulation and observed statistics, as the peaks in both distributions rotate as a function of the IMF clock angle. However, it is necessary to take into account the modulation of the statistical distribution that is caused by a bias towards in situ FTE signatures being observed in the winter hemisphere (an effect that has previously been predicted and observed in this data set). Taking this seasonal effect into account, the FTE locations support the previous simulation results and confirm the earlier prediction that the energy transfers in the plane of the IMF. In addition, we investigate the effect of the dipole orientation (both the dipole tilt angle and its orientation in the plane perpendicular to the solar wind flow) on the energy transfer spatial distribution. We find that the energy transfer occurs mainly in the summer hemisphere, and that the dayside reconnection region is located asymmetrically about the subsolar position. Finally, we find that the energy transfer is $10 \%$ larger at equinox conditions than at solstice, contributing to the discussion concerning the semiannual variation of magnetospheric dynamics (known as "the Russell-McPherron effect").
\end{abstract}

Keywords. Magnetospheric physics (Magnetopause, cusp, and boundary layers; Solar wind-magnetosphere interactions)

\section{Introduction}

In recent years, research in space physics has been moving from a phenomenological towards a more quantitative description of the near-Earth plasma system. The main idea concerning the overall dynamics of the magnetosphere was sketched by Dungey (1961), who suggested that the interplanetary magnetic field (IMF) reconnects with the magnetospheric field at the dayside magnetopause, setting up convection of plasma and magnetic flux within the magnetosphere and ionosphere. Later, it became clear that reconnection indeed is the main controlling factor of the magnetospheric dynamics (e.g. Gonzalez and Mozer, 1974), especially when low latitude reconnection occurs (Nishida, 1983, and references therein). As in any physical system, a quantitative description requires information on the system energetics, and one of the first attempts in this direction was by Akasofu (1981), who established an $\epsilon$ parameter characterizing the magnetospheric energy input as a function of solar wind parameters.

Magnetospheric energy transfer is widely accepted to occur as a load-generator process (Siscoe and Cummings, 1969; Lundin and Evans, 1985). In this process, low latitude reconnection between the IMF and the magnetospheric magnetic field reconfigures magnetic flux in the dayside boundary layers, accelerating plasma and magnetic field lines. The magnetic flux opened by dayside reconnection is carried towards the tail lobes, initially by the release of magnetic tension and subsequently by the force exerted by the 
magnetosheath flow. In light of the energetics of the system, the dayside acts as a load, where energy is converted from magnetic into kinetic form. In the tail lobes, energy is extracted from the magnetosheath flow and energy is converted from kinetic form into magnetic form in a tail lobe generator. The first attempt to quantify the energy conversion process was made by Laitinen et al. (2006), who used a global MHD simulation to infer energy conversion surface density. They found that in the simulation, the dayside load indeed appears adjacent to the reconnection region, while the generators are located behind the cusps in the tail lobes. Rosenqvist et al. (2006) presented a method to investigate energy conversion using Cluster observations, and later the method was applied to both the load and generator cases (Rosenquist et al., 2008). The most recent step using this methodology was to characterize the load-generator process systematically in a small data set using Cluster observations (Anekallu et al., 2011). It was found that the sign of the energy conversion, indicating whether the magnetopause crossing occurs in a load or in a generator region, is sometimes ambiguous. While this was found to be mainly due to observational difficulties in determining the magnetopause current density, it also suggests that the load and generator regions may move on the magnetopause surface in a manner that has not been observationally investigated in detail.

Global MHD simulations have proved to be a useful tool for investigating magnetospheric energetics. Palmroth et al. (2003) introduced a method for investigating energy transfer within the magnetosphere using the GUMICS-4 simulation. They found that the magnetospheric energy transfer is controlled by the focussing of Poynting flux (Papadopoulos et al., 1999), where energy transfers in sectors perpendicular to the dayside reconnection line. While the phenomenon itself is a simple consequence of the Poynting theorem, it suggests that the magnetopause energy transfer has a spatial variation controlled by the IMF direction. Indeed, Palmroth et al. (2006) found that when the IMF is duskward and southward, energy transfers through the northern prenoon and southern postnoon lobes, whilst the energy transfers in the northern postnoon and southern prenoon lobes for dawnward and southward IMF orientations. The first indications that the simulation energy transfer patterns at the magnetopause were supported by observational evidence were presented by Palmroth et al. (2011), who found that in two carefully selected events the simulation results were in qualitative and near-quantitative agreement with Cluster observations. However, apart from these two cases, the spatial variation of energy transfer at the magnetopause, as controlled by the IMF orientation, has not been systematically studied.

A major manifestation of the reconnection process (and hence energy transfer) at the magnetopause is the flux transfer event, or FTE (Russell and Elphic, 1978, 1979). FTEs occur when the reconnection process switches on and then off again, or when the reconnection rate enhances and then decreases. This process opens magnetic flux which moves away from the reconnection site under the influence of the magnetosheath flow and magnetic tension. The magnetic structure formed by reconnection moves past a spacecraft situated near the magnetopause, giving rise to distinctive signatures. The most noticeable signature is a bipolar variation in $B_{\mathrm{n}}$, the component of the magnetic field that is normal to the magnetopause (Russell and Elphic, 1978). If the signature is a so-called "standard polarity" signature (i.e. $B_{\mathrm{N}}$ undergoes a positive-then-negative variation), this indicates that the FTE structure is moving broadly northward, whereas a "reverse polarity" negative-then-positive signature (Rijnbeek et al., 1982) indicates southward motion. Several models have been put forward to explain the signatures, most of which are based on reconnection. In these models, the structure takes the form of a flux rope (Russell and Elphic, 1978; Lee and $\mathrm{Fu}, 1985$ ) or a thickening of the magnetopause boundary layer (Southwood et al., 1988; Scholer, 1988). (See Sonnerup, 1988; Lockwood and Hapgood, 1998, or Fear et al., 2008, 2010a,b, for further discussion of the differences between these conceptual models.)

Reconnection need not occur as a bursty process, and if it takes place at a steady rate then FTE signatures will not be observed. However, Saunders et al. (1984) and Milan et al. (2000) have estimated that FTEs may make a significant contribution to the transport of flux within the magnetosphere, so in this study we make use of the occurrence of FTE signatures as a proxy for energy transfer. Accordingly, we seek to compare the location of FTE signatures for various IMF orientations with the locations of energy transfer in the GUMICS-4 model. If FTEs are formed by a low latitude reconnection site whose orientation tilts in response to variations in the IMF $B_{\mathrm{y}}$ component (Gonzalez and Mozer, 1974), then the division between standard and reverse polarity events (indicating northward and southward motion respectively) should also rotate in response to changes in $B_{\mathrm{y}}$. Previous studies had found that the spatial distribution of FTE polarities was not simply ordered by the IMF direction (Berchem and Russell, 1984), although Russell et al. (1985), using the same dataset, found that if the polarity information was discarded and FTEs which occurred when the IMF had a dawnward orientation were combined with those which occurred when the IMF was duskward (by reflecting the location of dawnward IMF events about the noon meridian) then the spatial distribution was suggestive of a low latitude component reconnection site. When the IMF was strongly dawnward or duskward (within $30^{\circ}$ of the GSM equator) the FTE signatures were aligned in a dawn-dusk band; when $\left|B_{\mathrm{y}}\right|$ and $\left|B_{Z}\right|$ were approximately equal, then the FTE signatures were arranged in a band which was tilted with respect to the equator. When the IMF was strongly southward, FTE signatures were observed over a large part of the magnetopause.

Daly et al. (1984) found that the division between the location of magnetosheath FTE signatures with energetic ion anisotropies antiparallel and parallel to the magnetic field (indicating a connection to the Northern and Southern 
Hemispheres, respectively) was inclined in opposite directions for positive and negative IMF $B_{\mathrm{y}}$ conditions, in a manner consistent with division similar to tilted subsolar reconnection line (Gonzalez and Mozer, 1974). Kawano and Russell $(1996,1997)$ investigated the local time distribution of the "rotational polarity" of FTE signatures. The rotational polarity (right-handed or left-handed) gives the direction of the rotation of the magnetic field within the MN plane (the plane perpendicular to the projection of the Earth's dipole onto the local magnetopause surface). It is used as a proxy for the direction of azimuthal motion of the FTE (dawnward or duskward). Kawano and Russell $(1996,1997)$ found that the rotational polarity was generally left-handed pre-noon and right-handed post-noon, consistent with generally antisunward motion of FTEs. However, rotational polarities indicative of Sunward motion were observed in the northern pre-noon and southern post-noon quadrants when the IMF $B_{\mathrm{y}}$ component was negative, and in the northern post-noon and southern pre-noon quadrants when $B_{\mathrm{y}}$ was positive. The locations of these Sunward moving events were also indicative of FTEs being formed at a tilting subsolar component reconnection line as proposed by Gonzalez and Mozer (1974).

In addition to previous work concerning the spatial occurrence of FTEs, Raeder (2006) predicted that a seasonal bias should be present in FTE statistics. In his simulations, FTEs were formed by multiple X-line reconnection, and only occurred in solstice conditions when the dipole tilt $\Psi$ in the $\mathrm{xz}$ plane was non-zero. When this was the case, $\mathrm{X}$-lines were formed sequentially with one situated near the equator and another displaced into the winter hemisphere. The subsequent motion of most FTEs was towards the winter hemisphere. There is observational evidence from both INTERBALL and Cluster that this effect is indeed present at the magnetopause to some degree (Korotova et al., 2008; Fear et al., 2012), although Fear et al. (2012) found that in situ FTE signatures were also observed near equinox, contrary to the Raeder (2006) prediction. If this seasonal bias is present, then this introduces a limit to the use of FTE signatures as a proxy for energy transfer. In the multiple X-line model, most of the open flux (and hence energy) is transferred by magnetic field lines that do not map through the flux rope (Fear et al., 2008). Therefore, if FTEs are mostly formed by multiple $\mathrm{X}$-line reconnection, then the location statistics could be used to indicate to some extent where on the magnetopause energy is being transferred, but not necessarily to compare between hemispheres, or to compare between significantly different local times. (Due to orbital precession, a given spacecraft will sample different parts of the magnetopause during different terrestrial seasons.)

Here we investigate whether it is possible to validate the energy transfer results from simulations using the statistical occurrence of FTE signatures. First, we investigate the rotation of the energy transfer pattern at the magnetopause as a response to IMF $B_{\mathrm{y}}$ by using the statistical occurrence of FTEs as an observational means to validate simulation re- sults. Second, since the location of the FTE signatures appears to depend upon the dipole tilt (Raeder, 2006; Korotova et al., 2008; Fear et al., 2012), we also investigate the dipole tilt dependence of the energy transfer in the GUMICS-4 simulation which, to our knowledge, has not been examined before. The paper is organized as follows: first we describe the GUMICS-4 global MHD simulation and the FTE data set. Second, we present the comparisons of the simulation results with the FTE statistics concerning the IMF $B_{\mathrm{y}}$. Then we consider the tilt angle dependence in the GUMICS simulation, and we end the paper with discussion and conclusions. The geomagnetic solar magnetospheric (GSM) coordinate system is used to illustrate the effect of the IMF $B_{\mathrm{y}}$ component on the FTE distribution and the GUMICS energy transfer, while the geomagnetic solar ecliptic (GSE) is used to depict the effect of the dipole tilt effect on energy transfer.

\section{Data and methods}

GUMICS-4 (Janhunen, 1996) is a global magnetohydrodynamic (MHD) simulation of the coupled solar windmagnetosphere-ionosphere system. GUMICS-4 solves the fully conservative MHD equations within the simulation box including the solar wind and the magnetosphere, extending from $+32 R_{\mathrm{E}}$ to $-224 R_{\mathrm{E}}$ in $\mathrm{x}$-direction and $\pm 64 R_{\mathrm{E}}$ in the yz-directions. The MHD simulation box is coupled to another simulation domain representing the ionosphere, where electrostatic equations hold. The coupling is carried out via field-aligned currents and precipitation from the magnetosphere, and by the electric potential which is computed within the ionosphere and passed to the magnetosphere where it is used as a boundary condition. Other boundary conditions include the solar wind parameters within the Sunward wall of the simulation box, and the Earth's dipole field. The solar wind parameters can be given from an event observed with an upstream spacecraft, or artificial synthetic solar wind data can be used to investigate the code behavior in simplified conditions. The full set of plasma parameters in space and time are given as an output of the computations. GUMICS-4 uses a cell-by-cell adaptive grid, allowing finer resolution at locations of large spatial gradients; on the other hand, a coarser grid can be used in less important places.

The Palmroth et al. (2003) method quantifies energy transfer by searching the magnetopause surface from the GUMICS-4 simulation from each saved file, giving the magnetopause location as a function of time. The magnetopause is essentially defined as a void in the solar wind streamlines encompassing the magnetosphere. Other codes have used this method (Shukhtina et al., 2009), indicating that the method is robust and gives a smooth magnetopause location as compared with, for example, gradient-based methods. Once the location of the magnetopause is known, the total energy density vector at the magnetopause location can be extracted. In practice, the energy flows towards the 


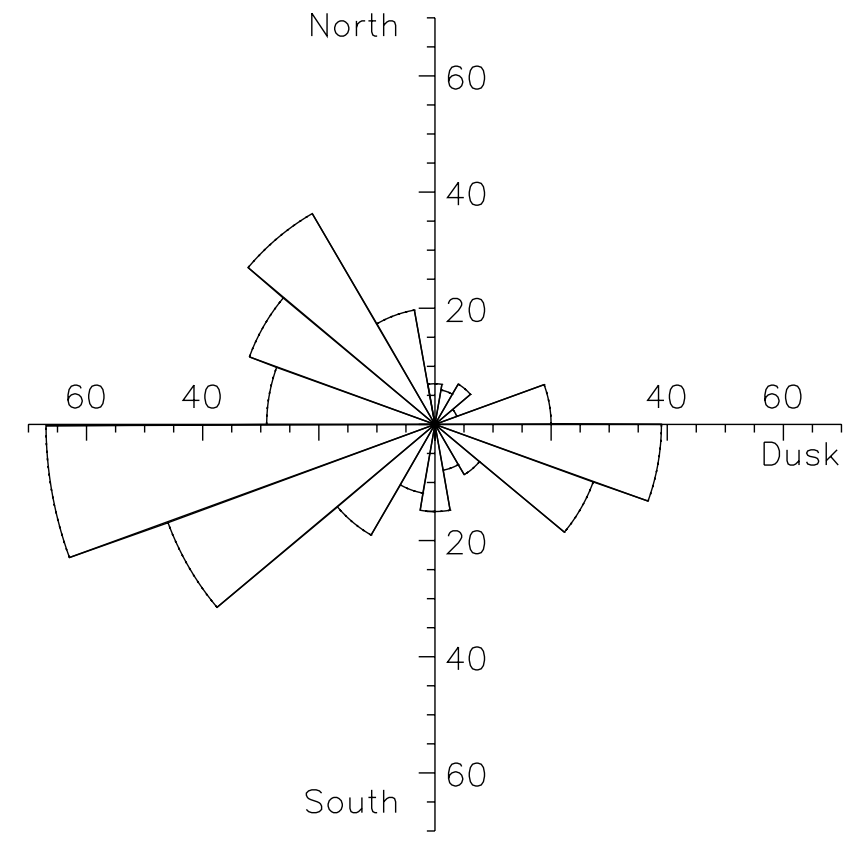

Fig. 1. The IMF clock angle distribution of the FTEs in the Fear et al. (2005) data set. The length of each bar indicates the number of events in each IMF direction. (Reproduced from Fear et al., 2007.)

magnetopause in most places (Palmroth et al., 2006), making the net energy transfer from the solar wind into the magnetosphere. The details of the method as well as the various ways to utilize the results are presented in Palmroth et al. (2011).

Fear et al. $(2005,2007)$ investigated the occurrence of FTEs from Cluster observations, and it is their dataset that we shall use in this paper to compare with the GUMICS-4 simulation. The authors used data from the Cluster FGM (Balogh et al., 2001) instrument to identify 446 FTE signatures which were observed by Cluster between November 2002 and June 2003, by looking for a bipolar signature in the magnetic field component normal to the magnetopause. During this time, the orbit of the Cluster spacecraft precessed such that it crossed the post-terminator magnetopause on the dusk flank (November), through the post-noon sector until it observed the near-noon magnetopause (February/March), then the prenoon magnetopause and finally the post-terminator magnetopause on the dawn flank (June). When the orbit crossed the magnetopause on the flanks, the magnetopause crossings occurred near apogee and so occurred near the equator; on the other hand, due to the high inclination of the Cluster spacecraft the magnetopause crossings nearer noon occurred at higher latitudes. Recently, Fear et al. (2012) presented a further analysis of this dataset, examining the relationship between the location at which FTE signatures are observed and the IMF $B_{\mathrm{y}}$ component. They found that the location of standard and reverse polarity FTE signatures was generally well ordered by the IMF orientation, but that this effect was complicated by the seasonal modulation predicted by Raeder
(2006). Fear et al. (2012) found that FTEs were observed preferentially in the Northern Hemisphere post-noon and Southern Hemisphere pre-noon. Since Cluster observed the post-noon magnetopause in the Northern Hemisphere winter (November 2002-February 2003) and the pre-noon magnetopause mostly during Southern Hemisphere winter (MarchJune 2003), this trend is consistent with the bias towards the winter hemisphere that was predicted by Raeder (2006).

Figure 1 shows a polar histogram of the time-lagged IMF clock angle at the time of each FTE $\left(\theta=\tan ^{-1}\left(B_{\mathrm{y}} / B_{\mathrm{z}}\right)\right)$, reproduced from Fear et al. (2007). Clearly, the FTEs are observed most commonly during equatorial IMF. This is in part consistent with the hypothesis that the FTE spatial distribution depends on the IMF orientation; when the IMF is equatorial, FTE signatures should be observed mainly on the dawn and dusk flanks, which are regions in which the spacecraft "skimmed" the magnetopause for hours at a time, and therefore was more likely to observe FTE signatures (Fear et al., 2007). Note that more FTE signatures were observed when the IMF was southward and dawnward than when it was southward and duskward. This is in part because the IMF was more likely to have a southward/dawnward orientation than a southward/duskward orientation during the interval that was surveyed (Fear et al., 2007), but it is also because Cluster observed the post-noon Northern Hemisphere magnetopause and pre-noon Southern Hemisphere magnetopause (the most likely locations for FTE signatures when the IMF is southward and dawnward) during the local hemisphere's winter (Fear et al., 2012). The events which occur when the IMF is strongly northward are observed at the postterminator magnetopause, and are consistent with an origin at a high latitude reconnection site (Fear et al., 2005). Since they are not attributable to low latitude reconnection, they shall not be considered in this study.

\subsection{IMF $B_{\mathrm{y}}$ dependence of energy transfer}

The GSM coordinate system is used throughout this section. Figure 2 illustrates the general features of energy transfer through the simulation magnetopause, as computed using the method described by Palmroth et al. (2003). While Fig. 2 shows the results from a synthetic run where the IMF clock angle is rotated from north to dusk to south and to dawn in six hours, similar features are observed from other types of simulations as well (Palmroth et al., 2011). The magnetic field intensity in the run is $5 \mathrm{nT}$, while the solar wind dynamic pressure is $2 \mathrm{nPa}$ and the dipole tilt angle is set to zero. The legend in each distribution gives the total net energy transfer extending from the nose of the magnetopause to the tail at $X_{\mathrm{GSM}}=-30 R_{\mathrm{E}}$. Each bar in the diagram gives the amount of energy transferred in that particular direction in the yz plane (again, from the nose to $30 R_{\mathrm{E}}$ downtail), viewed tailward from the front of the magnetopause. Hence, each panel illustrates the spatial variation of the amount of energy transfer at the magnetopause for a different IMF 
(a)

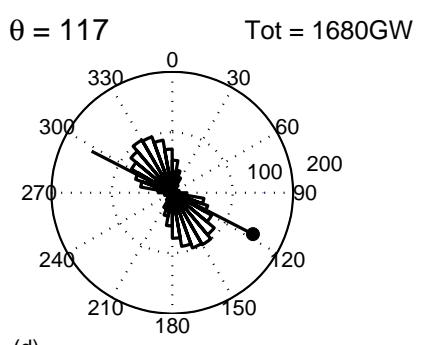

(d)

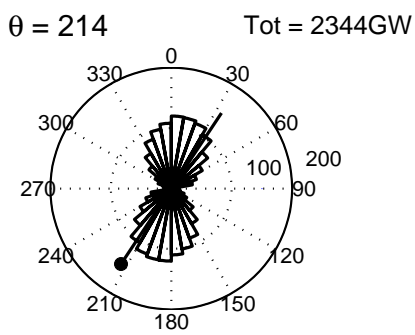

(b)

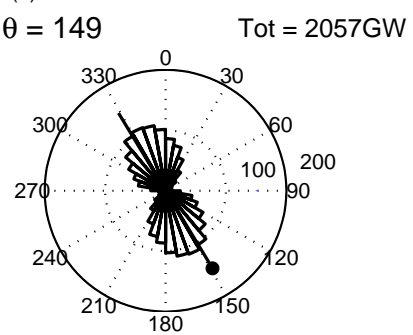

(e)

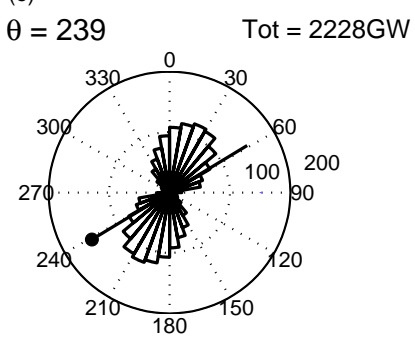

(c)

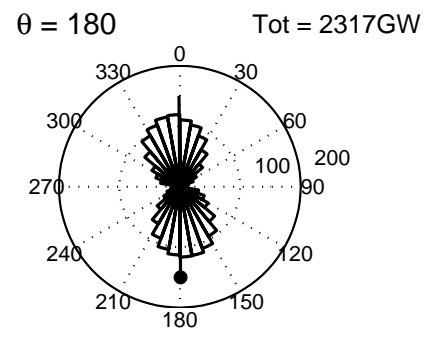

(f)

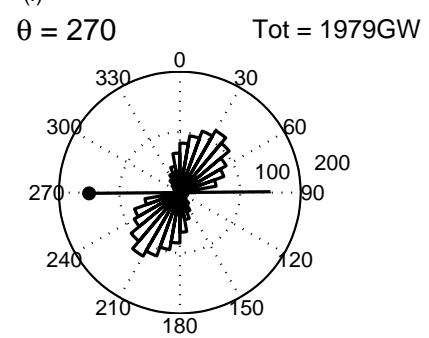

Fig. 2. (a)-(f) The distributions of energy transfer in the GUMICS-4 simulation, for different clock angles $(\theta)$. The clock angle is indicated by the black line and given in the legend. The energy transfer distributions are normalized to the outer circle (200GW), and the total net energy transfer for each time instant is also given in the legend.

orientation. When the IMF is strongly southward, energy transfers roughly in the noon-midnight meridian in both the Northern and Southern Hemisphere. When $B_{\mathrm{y}}$ is positive, energy transfers in the northern pre-noon and southern postnoon quadrants; and vice versa when $B_{\mathrm{y}}$ is negative.

Figure 3 shows the locations of the FTE signatures at the magnetopause which occurred when the IMF was southward and dawnward (Fig. 3a-b) and southward and duskward (Fig. 3c-d). Panels (a) and (c) show the locations at which standard polarity signatures were observed (indicating a broadly northward motion) and panels (c) and (d) show the locations at which reverse polarity (southward-moving) signatures were observed. Clearly, for dawnward and southward IMF, the FTEs preferably occur in the northern post-noon and southern pre-noon sectors at the magnetopause. This is similar to the clockwise rotation of the GUMICS-4 energy transfer distribution in Fig. 2e and $\mathrm{f}$ which occurs when $B_{\mathrm{y}}$ is negative. When the IMF is duskward and southward, the FTE locations are not as clearly rotated about the noon-midnight meridian as in the simulation results due to the absence of events during the local hemisphere summer. However, the standard polarity FTE distribution is rotated anticlockwise to some extent with respect to the standard polarity FTE distribution when $B_{\mathrm{y}}$ is negative. There are no reverse polarity FTE signatures in the southern post-noon quarters of Fig. 3b and d, again due to the seasonal effect. (For a more detailed discussion of these points, see Fear et al., 2012.)

To investigate the simulation results during actual event conditions and not during artificial simplified solar wind conditions, we investigated the spatial variation of the energy transfer in eleven separate events listed in Table 1, repre-
Table 1. Events for the energy transfer spatial dependence analysis.

\begin{tabular}{ll}
\hline Date and time (UT) & Type \\
\hline 1998: 28 Mar, 22:00-29 Mar, 07:00 & substorm \\
1999: 23 Nov, 12:00-15:00 & steady convection \\
2001: 16 Jan, 21:00-23:55 & steady solar wind \\
2001: 26 Jan, 08:00-12:00 & steady solar wind \\
2001: 15 Aug, 03:00-10:00 & substorm \\
2001: 21 Aug, 08:35-15:00 & substorm \\
2001: 8 Sep, 18:00-23:00 & substorm \\
2004: 18 Feb, 15:00-23:55 & substorm \\
2005: 21 May, 04:00-10:00 & steady convection \\
2005: 3 Dec, 17:00-22:00 & substorm \\
2005: 28 Dec, 19:35-23:55 & substorm \\
\hline
\end{tabular}

senting 722 time instants and $60 \mathrm{~h}$ of simulated periods at which the energy analysis is carried out and solar wind conditions are known. This constitutes the largest semi-statistical energy transfer data set from simulation results, and is the data set that we shall compare with the FTE statistics. Table 1 shows that most of the simulated periods include substorms, but there are two periods with steady solar wind conditions, and two events of the "steady convection" type, during which ionospheric convection is in progress but there are no particular sudden increases or decreases in the $\mathrm{AE}$ index. Hence, although substorms appear to be the most commonly investigated feature in the runs, the time instants include all kinds of solar wind conditions (see, e.g. Honkonen et al., 2011), and hence the results will not only be 

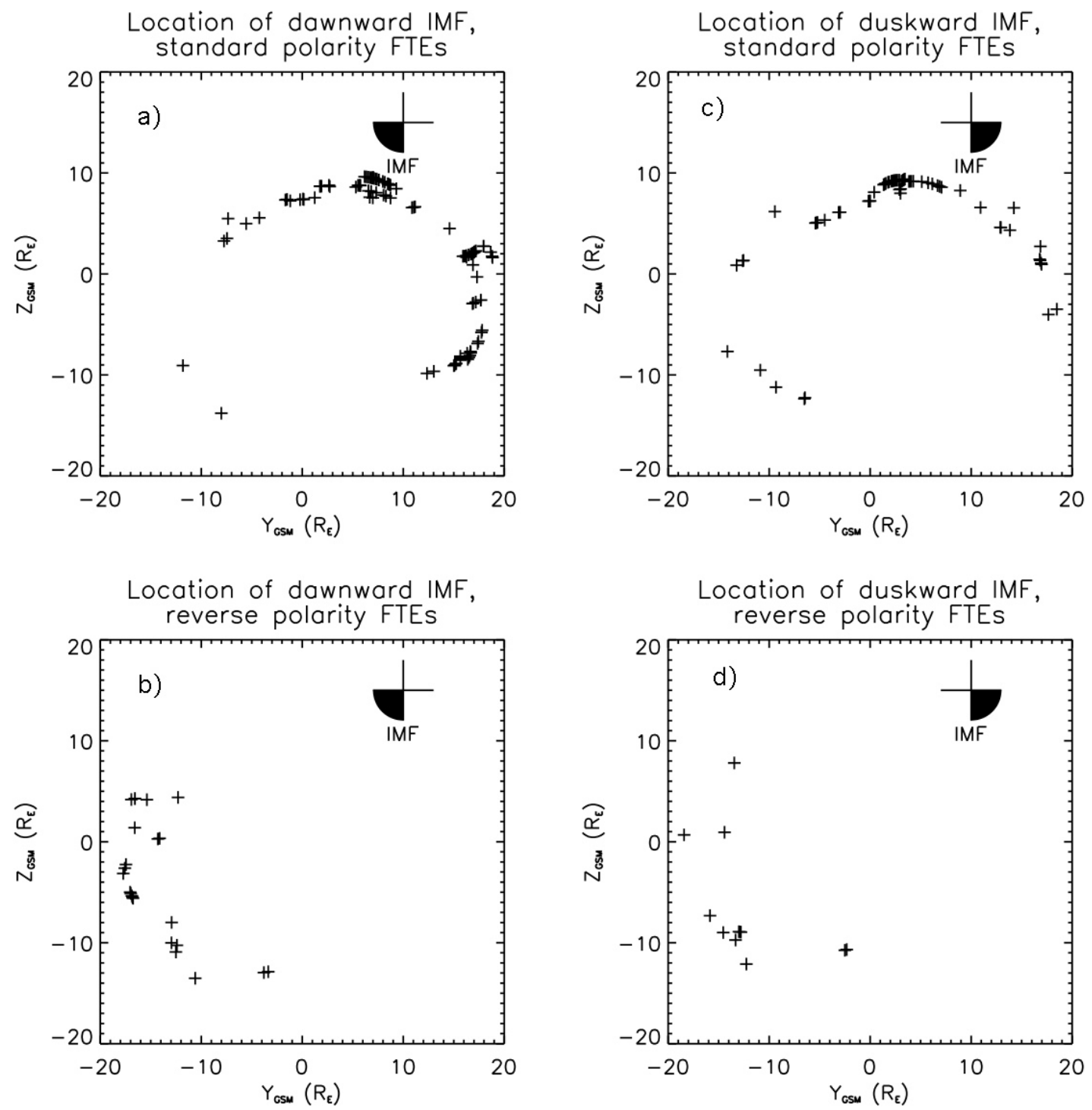

Fig. 3. Locations of the FTEs binned by the sign of the IMF GSM $B_{y}$ component. Locations of (a) standard and (b) reverse polarity FTEs which occurred when the IMF was southward and dawnward, and the locations of (c) standard polarity and d) reverse polarity FTEs which occurred when the IMF was southward and duskward. North is at the top, dusk to the right, south at the bottom, and dawn to the left.

applicable to substorms. Five of the events occur during Northern Hemisphere winter (December-February), one during spring (March-May), two in summer (July-August), and two during the fall (September-November). Figure 4 shows the IMF clock angle directions during the events listed in Table 1 . The time instants in our data set cover a broad range of southward and IMF $B_{\mathrm{y}}$-dominated IMF orientations, allowing a valid comparison between this data set and the FTE statistics.

Figure 5 shows a comparison of the simulation results from events listed in Table 1 against the FTE data set. Both the FTE data set and the simulation results are presented in the GSM coordinate system. Figure 5 shows eight clock angle ranges in different panels. In each panel the leftmost di- agram shows the clock angle range for which the other diagrams are plotted. The center diagram in each panel shows the average energy transfer distribution in the events in Table 1 during the clock angle range in question. The length of each bar indicates the amount of energy transferred through the magnetopause in each specific location and the red line is a component reconnection line calculated using Eq. (1) of Gonzalez and Mozer (1974) (assuming geomagnetic and magnetosheath magnetic field strengths of $50 \mathrm{nT}$ and $30 \mathrm{nT}$, respectively). The shaded part of the center distribution corresponds to the part of the magnetopause which Cluster observes northward of the $\mathrm{X}$-line and at $Y_{\mathrm{GSM}}>0$ (which corresponds to Northern Hemisphere crossings during the Northern Hemisphere winter, since the intersection of the Cluster 


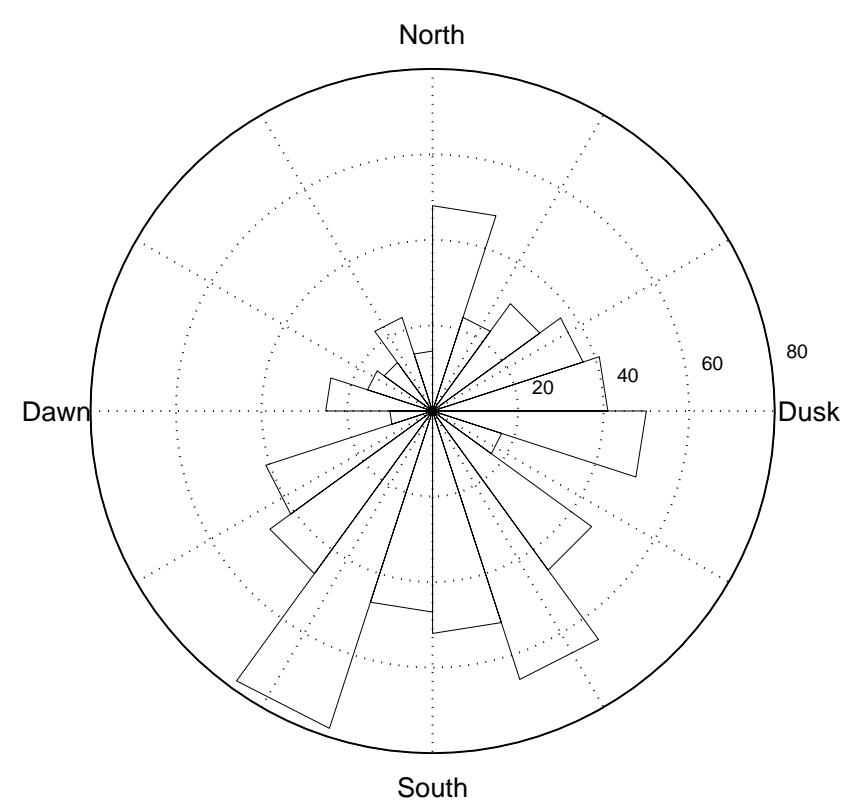

Fig. 4. The IMF clock angle distribution for all events simulated with GUMICS-4, listed in Table 1. The bar size indicates the number of time instants during the simulations during which the clock angle was in the specified direction in GSM coordinate system. North is at the top, dusk to the right, south at the bottom, and dawn to the left.

orbit and the Northern Hemisphere magnetopause crosses the GSM YZ plane in March). Hence the shaded part illustrates the part of the energy transfer distribution that we expect to observe in the Cluster FTE statistics, taking into account the combined effect of the seasonal bias predicted by Raeder (2006) and the orbital bias of the spacecraft. The rightmost diagram shows a polar histogram of the locations of standard polarity FTE signatures that were observed during the corresponding IMF conditions (taken from Fear et al., 2012). The red arrow in each polar histogram shows the average location of the FTEs. The number of events in each clock angle bin varies; Fig. 5d-f each contain fewer than 15 FTEs, and so the statistical significance is weakest in these panels. (Due to the small number of reverse polarity signatures in most of these clock angle bins, we only consider standard polarity signatures in this analysis.)

Figure 5 indicates that while the correspondence of the two data sets is not perfect, there is a qualitative agreement between the FTE statistics and the shaded part of the GUMICS4 distribution (which indicates the areas of the magnetopause that are expected to compare well given the seasonal effect that is present in the spacecraft observations, and the orbital bias of Cluster). In Fig. 5a, the observed FTE distribution peaks clockwise of the Northern Hemisphere peak in the GUMICS-4 energy transfer. Both peaks rotate weakly anticlockwise in Fig. 5b-c. In Fig. 5d and e, the correspondence is not so good; however, the strongly southward
IMF bins are the least populous in terms of FTE statistics. In Fig. 5f, the correspondence is again not so good, with several FTEs observed just northward of the GSM equator, compared with the shaded part of the GUMICS distribution which is more tightly aligned with the vertical axis. Nevertheless, the correspondence improves significantly in Fig. $5 \mathrm{~g}$ and 5h, where both the FTE location statistics and the part of the energy transfer distribution that corresponds to the magnetopause observed by Cluster during Northern Hemisphere winter peak immediately post-noon, with very little scatter. Since the Northern Hemisphere peaks of the observed FTE and model energy transfer distributions are both in the northern post-noon quadrant when the IMF is dawnward and slightly northward (Fig. 5a), rotate anticlockwise as the IMF vector also rotates anticlockwise and (when the part of the energy transfer distribution that would be observed when Cluster is in the Northern Hemisphere summer is curtailed) both lie immediately post-noon when the IMF is strongly duskward (Fig. 5g and h), we take this as evidence that the energy transfer distribution at the magnetopause does indeed depend upon the IMF clock angle in this manner. However, the correspondence between the two distributions is not exact, as the FTE distribution peaks clockwise of the majority of the Northern Hemisphere energy transfer distribution when the IMF has a significant negative $B_{\mathrm{y}}$ component (Fig. 5a-c), indicating that rotations in the FTE distribution are perhaps more pronounced than those in the simulation energy transfer distribution. The necessity of curtailing the part of the distribution relating to the pre-noon Northern Hemisphere magnetopause (observed by Cluster during the Northern Hemisphere summer) is interpreted as a direct consequence of the FTE seasonal effect predicted by Raeder (2006), and will be discussed further in the Discussion.

\subsection{Dipole tilt dependence of energy transfer}

To investigate the imbalance of the energy transfer distribution between the Northern and Southern Hemisphere that is visible in the simulation results in Fig. 5e-g, we show results from five additional runs in which we varied the dipole tilt angle in Fig. 6. In this section we use the geomagnetic solar ecliptic (GSE) coordinate system as it emphasizes the effect of the dipole tilt angle in the yz plane. Each diagram in Fig. 6a-e illustrates one time instant after a prolonged period of strictly southward IMF, while the other solar wind conditions are kept constant. The time history for all time instants is the same, while the dipole is varied to represent different times of year. The magnitude of the tilt angle ranges from $-23^{\circ}$ to $+23^{\circ}$. Clearly, for the Northern Hemisphere winter (Fig. 6a) the energy transfer occurs preferably in the Southern Hemisphere. Towards the equinox (Fig. 6b), the imbalance between the Northern and Southern Hemisphere becomes less pronounced, and at equinox (Fig. 6c) the amount of energy transferring in the Northern and Southern Hemisphere is the same. Towards the northern 

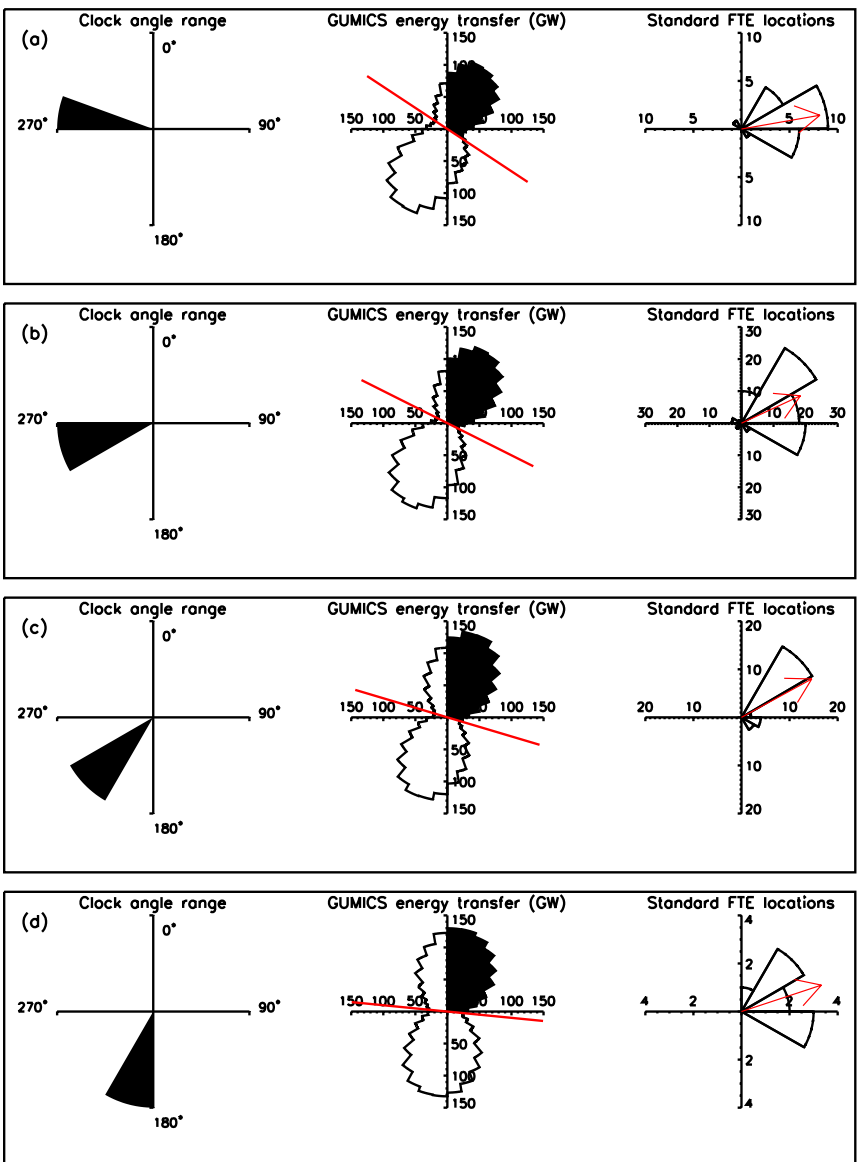
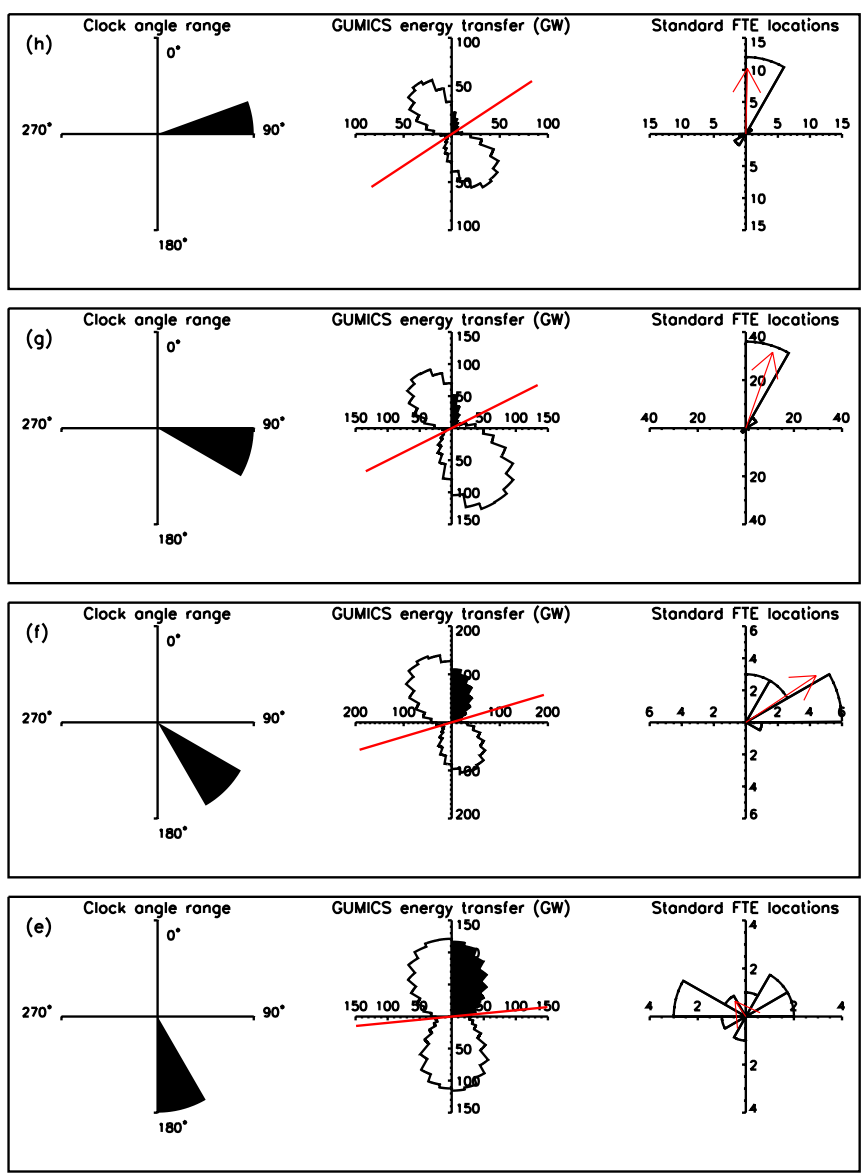

Fig. 5. Comparison of the FTE locations against the GUMICS-4 energy transfer as a function of IMF clock angle. In each panel, the leftmost diagram shows the clock angle range for which the two other diagrams are plotted. The center diagram shows the average energy transfer distribution which occurs in the GUMICS-4 model during the events listed in Table 1, projected onto the GSM yz plane. The red line is a component reconnection line calculated using Eq. (1) of Gonzalez and Mozer (1974) (assuming geomagnetic and magnetosheath magnetic field strengths of $50 \mathrm{nT}$ and $30 \mathrm{nT}$, respectively). The shaded part of the center distribution corresponds to the part of the magnetopause which Cluster observes northward of the X-line during the Northern Hemisphere winter. The rightmost diagram is a polar histogram of the standard polarity FTE locations with the red arrow indicating the average direction of motion (Fear et al., 2012). Note that the scales in each diagram are not the same.

summer, the energy transfer starts to occur more in the Northern Hemisphere (Fig. 6d and e). The total amount of energy transfer, shown in the legend below each panel, shows that the maximum energy transfer in the GUMICS simulation occurs near equinox, whilst the total amount of energy transfer is reduced by about $10 \%$ near solstice.

The color-coding in Fig. 7 shows the energy conversion surface density at the magnetopause (Laitinen et al., 2006) during the time instants in Fig. 6. The energy conversion surface density illustrates how much energy is converted between the kinetic and magnetic forms and is equivalent to the negative of the Poynting flux divergence. Positive (red) values around the subsolar position indicate the reconnection region and the region where magnetic tension in the newlyopened magnetic field lines is subsequently released. The positive values indicate that the Poynting flux divergence is negative, meaning that the energy converts from magnetic to kinetic form (Laitinen et al., 2006). The tail region is shown by negative (blue) values, indicating that the energy converts from kinetic to magnetic form. While the energy transfer (Palmroth et al., 2003) and the energy conversion (Laitinen et al., 2006) are two different issues, they both represent features of subsolar reconnection and are calculated from the same parameters. Hence, the integration of the energy transfer shown in Fig. 6 is in good agreement with the spatial values in Fig. 7. The magnitude of the energy conversion (the blue and red regions in Fig. 7) show a clear tilt-dependence, with a larger energy conversion from kinetic to magnetic form occurring in the summer hemisphere. This is consistent with the magnitude of energy transfer shown in Fig. 6. Altogether, the energy conversion at the magnetopause in the GUMICS model shows a clear inter-hemispheric difference which depends upon the tilt angle. 


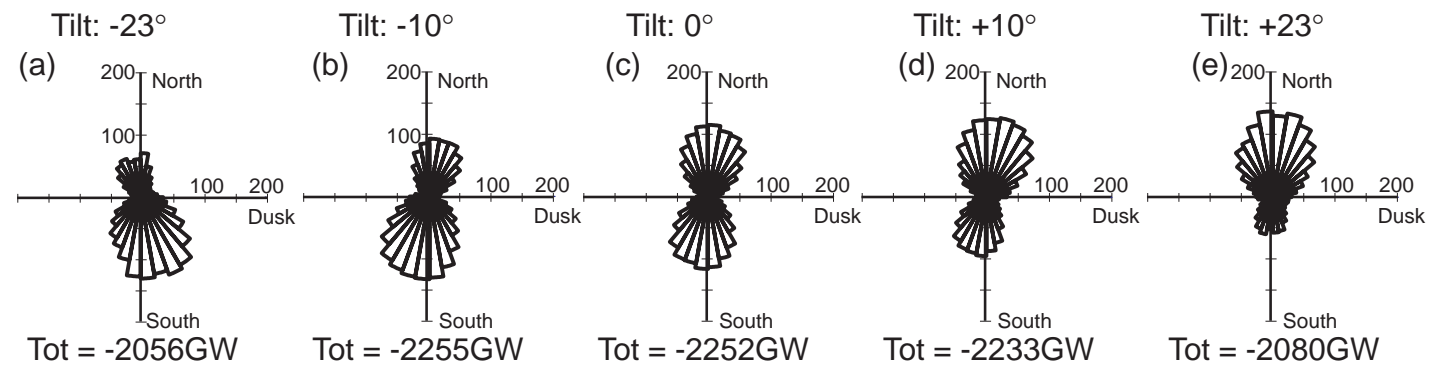

Fig. 6. Energy transfer spatial distribution at the magnetopause in the GSE coordinate system for dipole tilt angles (a) $-23^{\circ},(\mathbf{b})-10^{\circ}$, (c) $0^{\circ}$, (d) $+10^{\circ}$, and (e) $+23^{\circ}$. The bar size indicates the integrated amount of energy transfer in the specific location at the magnetopause. All diagrams are taken from a run where the IMF has been southward for a long time, and the conditions before the shown time are otherwise identical. The total amount of energy transfer for each diagram (summing all bars together) is given below the diagram.

The region in which the sign changes from positive to negative energy conversion surface density represents the location at which the field line that has been opened by reconnection stops being pulled by magnetic tension and is carried tailwards entirely by the magnetosheath flow (acting against the magnetic tension). Figure 7 indicates that the location at which the sign changes depends on the sign of the dipole tilt angle. In the winter hemisphere, the conversion of energy from magnetic to kinetic dominates further towards the tail before energy starts to convert from kinetic to magnetic form. In the summer hemisphere, the tail generator area appears closer to the subsolar region. In zero dipole tilt conditions, the dayside load area is evenly distributed with respect to the subsolar point.

Figures 6 and 7 also show a rotation of the energy transfer/energy conversion distributions about the noon-midnight meridian, even though the runs are carried out using identical constant solar wind conditions with southward IMF in all of the runs. The black lines in Fig. 7a-e indicate the dipole axis orientations in the yz plane of the GSE coordinate system. Hence, we conclude that the energy transfer spatial variation at the magnetopause is also dependent on the dipole axis orientation in the plane perpendicular to the solar wind flow.

\section{Discussion}

In this paper we have investigated the spatial variation of magnetopause energy transfer as shown by the global MHD code GUMICS-4 and statistical results concerning FTEs. The main purpose of the paper is to investigate whether the FTEs, which are also energy transfer events, can be used as proxies to verify the spatial variation of energy transfer from a global MHD simulation. Our aim was twofold; on one hand we examined how well the previously recognized rotation of the energy transfer pattern (due to the IMF dawn-dusk component) compares with the locations of suitably binned set of FTE signatures. On the other hand, since the FTE signature locations include a dependency on the dipole tilt angle (Raeder, 2006), we examined the dipole tilt angle dependence in the magnetopause energy transfer, which has not been previously studied to the authors' knowledge. We find that the comparison between the FTE statistics and GUMICS-4 energy transfer is clearest when considering negative $B_{\mathrm{y}}$; the FTE distribution is rotated with respect of the noon midnight meridian such that the events are more frequently observed in the northern postnoon and southern prenoon. This verifies previous simulation results (e.g. Palmroth et al., 2006) suggesting that the energy transfer pattern at the magnetopause is dependent on IMF $B_{\mathrm{y}}$ and rotates in a similar manner to the FTEs shown here.

When the IMF $B_{\mathrm{y}}$ component is positive, we find that situation is more complicated, due to the reduced likelihood of observing in situ FTE signatures in the summer hemisphere. In their raw form, the simulation results are not as well supported by the FTE statistics as when $B_{\mathrm{y}}$ is negative. However, if we consider only the part of the GUMICS energy transfer distribution that corresponds to the post-noon Northern Hemisphere magnetopause (which Cluster observes during the Northern Hemisphere winter), then the correspondence is much better (Fig. 5g and h). In comparing observed FTE statistics with the energy transfer in the simulation, we make two points of caution. First, the FTE signatures are not the only component of the total energy transfer. If multiple $\mathrm{X}$ line reconnection is occurring, as implied by the observed seasonal dependency, the implication is that most energy is transferred by open field lines that are not part of the flux rope (Fear et al., 2008), and so do not give rise to in situ FTE signatures (unless the real situation is a combination of the single and multiple X-line scenarios put forward by Lee and Fu, 1985; Southwood et al., 1988; and Scholer, 1988). Therefore, although FTE signatures are useful indicators of energy transfer, there is a limit to their use in this manner. Secondly, since the seasonal effect appears to be present in the Cluster data set, the spacecraft are not in the pre-noon Northern Hemisphere at the right time of year to see flux ropes, which is why it is necessary to compare the statistics with only the shaded part of the energy distributions in Fig. 5. However, we would predict that FTEs would be observed in 

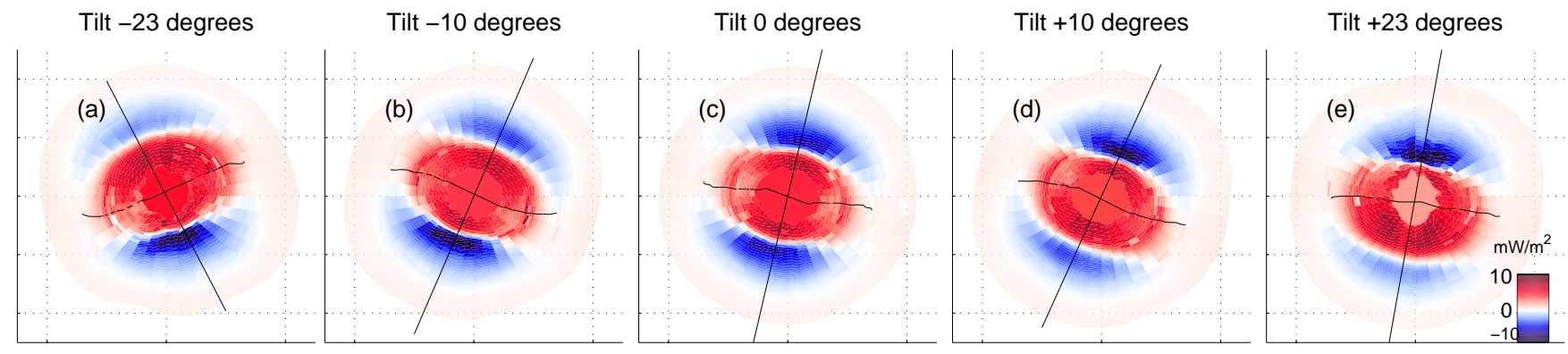

Fig. 7. Energy conversion surface density (Laitinen et al., 2006) in the GSE coordinate system, during the same times as presented in Fig. 6. The viewing angle is from the front of the magnetopause looking tailward, and the subsolar magnetopause is in the center of each diagram, while the the outer rim of the plotted surface is at $X_{\mathrm{GSE}}=-30 R_{\mathrm{E}}$. The straight vertical black lines indicate the dipole orientation in GSM yz plane, while the black curve through the subsolar position is the average of the reconnection line computed with the four-field junction method (Laitinen et al., 2006).

the sectors where the simulations suggest that energy is being transferred when $B_{\mathrm{y}}>0$, if a spacecraft were there during the Northern Hemisphere winter. Therefore, we do not believe that increasing the data set with further Cluster observations would help with validating the simulation results during positive IMF $B_{\mathrm{y}}$, because the seasonal bias would not change year-on-year. Hence a complete unbiased data set would require a survey of FTEs from other spacecraft, which is out of the scope of this paper.

The presented observations may also suggest that the energy transfer distribution is slightly broader than the simulation results imply. In Fig. 5a (where the FTE observations peak in the Northern winter Hemisphere, enabling the most direct comparison between the two data sets), the FTE distribution peaks at 80 degrees clockwise from the vertical axis, whereas the energy distribution peak is closer to 45 degrees. In Fig. 5g the FTE distribution peaks at about 20 degrees clockwise from the vertical axis and approximately 35 FTEs are observed between 0 and 30 degrees clockwise from the vertical axis (approximately $8 \%$ of the total number of FTEs observed). If the FTE distribution was simply the energy transfer distribution curtailed in the summer hemisphere, then from the energy distribution in Fig. $5 \mathrm{~g}$ we would expect a relatively small number of FTEs in that clock angle range. It is possible that the peak of the FTEs in the northern post-noon quadrant of Fig. $5 \mathrm{~g}$ and $\mathrm{h}$ could represent the flank of a distribution that is somewhat broader than the GUMICS energy transfer distribution (but which is curtailed pre-noon by the combination of the seasonal effect and orbital bias discussed above).

In examining the dipole tilt dependence of the energy transfer pattern at the magnetopause, we find that there is an imbalance of energy transfer according to the season in the simulation. In the Northern Hemisphere winter, the energy transfer occurs preferably in the Southern Hemisphere, and vice versa. There is also a $10 \%$ difference in the magnitude of the energy transfer; we find larger energy transfer values close to equinox compared with the values towards the solstices. The results of the simulations could therefore explain the observation that the magnetospheric activity increases at equinoxes (the "Russell-McPherron effect"; Russell and McPherron, 1973). The energy transfer values in the simulation are usually highly stable and repeatedly the same under similar conditions, and hence we regard the $10 \%$ difference as a real phenomenon produced by the physics in the simulation and not a numerical effect.

The opposite imbalance is present in the FTE statistics, but again we emphasize that there is a limit to the use of FTE statistics as a proxy for energy transfer. The simulation energy transfer and conversion are mostly dependent on the Chapman-Ferraro current density, which is larger within the hemisphere more directly exposed to the solar wind. Hence that hemisphere is logically converting more energy. On the other hand, if the FTEs are formed by multiple X-lines then most of the energy transfer takes place through open magnetic field lines which do not map through the flux rope. Hence we argue that while the FTE statistics can be used to validate the simulation energy transfer results in a general sense, there are also differences that appear due to the fact that the FTEs and the simulation energy transfer are not exactly comparable. To summarise, combining the results of the current paper with those of Raeder (2006), Korotova et al. (2008) and Fear et al. (2012), we would argue that most of the dayside flux ropes formed by reconnection move towards the winter hemisphere (providing some information on energy transfer), but that most energy transfer takes place on open field lines which do not map through the flux rope and are connected to the summer hemisphere.

According to the simulation results presented in this paper, the location where the dayside load region becomes the nightside generator also depends on the tilt angle, as the tail generator region appears closer to the subsolar region within the hemisphere that is transferring more power (the summer hemisphere). The energy transfer pattern also rotates in response to to the orientation of the dipole axis in the plane perpendicular to the solar wind flow. Palmroth et al. (2011) 
and Anekallu et al. (2011) found that the energy conversion estimates using spacecraft observations can have an ambiguous sign for several reasons, including the accuracy of the current density measurements. Our results indicate that in addition, the sign of the energy conversion at the spacecraft location can vary as the load and generator regions move about the subsolar point as a result of the dipole tilt angle and the orientation of the dipole axis in the plane perpendicular to the solar wind flow. Hence, our results must be taken into account when interpreting future spacecraft observations of energy transfer. We predict that single event observations of energy conversion using, for example, Cluster spacecraft crossings of the magnetopause can vary a great deal depending on the IMF, season, and the dipole axis orientation with respect to the solar wind flow. It is possible that other factors also contribute. For instance, the solar wind flow velocity in the $y$ - and z-directions influence the alignment of the magnetopause and the location at the magnetopause that is most exposed to the solar wind. We argue that it is really the geometric alignment of the magnetopause (which depends on the solar wind flow parameters) relative to the dipole axis orientation and the IMF direction that controls the location where, for example, a reconnection line forms.

In summary, we find that the spatial variation of energy transfer from the GUMICS-4 simulations is generally consistent with observed FTE statistics, but that in comparing the two data sets it is necessary to account for the fact that they are not measures of exactly the same thing. Since there is a tendency for FTE in situ signatures to be observed in the winter hemisphere (Raeder, 2006; Korotova et al., 2008; Fear et al., 2012), and Cluster samples the pre-noon Northern Hemisphere during the Northern Hemisphere summer, it is necessary to curtail the pre-noon part of the GUMICS energy transfer distribution. Once this is done, the comparison between the two data sets is generally good. Additionally, we find that the spatial variation of energy transfer also depends on the dipole tilt angle and its orientation with respect of the solar wind flow.

Acknowledgements. The research leading to these results has received funding from the European Research Council under the European Community's Seventh Framework Programme (FP7/20072013) / ERC Starting Grant agreement number 200141-QuESpace. The work of MP and IH is supported by the Academy of Finland, and RCF is supported by STFC grant ST/H002480/1.

Topical Editor I. A. Daglis thanks M. F. Marcucci and D. Sibeck for their help in evaluating this paper.

\section{References}

Akasofu, S.-I.: Energy coupling between the solar wind and the magnetosphere, Space Sci. Rev., 28, 121-190, 1981.

Anekallu, C. R., Palmroth, M., Pulkkinen, T. I., Haaland, S. E., Lucek, E. A., and Dandouras, I.: Energy conversion at the Earth's magnetopause using single and multispacecraft methods, J. Geophys. Res., 116, A11204, doi:10.1029/2011JA016783, 2011.
Balogh, A., Carr, C. M., Acuña, M. H., Dunlop, M. W., Beek, T. J., Brown, P., Fornacon, K.-H., Georgescu, E., Glassmeier, K.H., Harris, J., Musmann, G., Oddy, T., and Schwingenschuh, K.: The Cluster Magnetic Field Investigation: overview of in-flight performance and initial results, Ann. Geophys., 19, 1207-1217, doi:10.5194/angeo-19-1207-2001, 2001.

Berchem, J. and Russell, C. T.: Flux Transfer Events on the Magnetopause: Spatial Distribution and Controlling Factors, J. Geophys. Res., 89, 6689-6703, doi:10.1029/JA089iA08p06689, 1984.

Daly, P. W., Saunders, M. A., Rijnbeek, R. P., Sckopke, N., and Russell, C. T.: The Distribution of Reconnection Geometry in Flux Transfer Events Using Energetic Ion, Plasma and Magnetic Data, J. Geophys. Res., 89, 3843-3854, doi:10.1029/JA089iA06p03843, 1984.

Dungey, J. W.: Interplanetary field and the auroral zones, Phys. Res. Lett., 6, 47-48, 1961.

Fear, R. C., Fazakerley, A. N., Owen, C. J., and Lucek, E. A.: A survey of flux transfer events observed by Cluster during strongly northward IMF, Geophys. Res. Lett., 32, L18105, doi:10.1029/2005GL023811, 2005

Fear, R. C., Milan, S. E., Fazakerley, A. N., Owen, C. J., Asikainen, T., Taylor, M. G. G. T., Lucek, E. A., Rème, H., Dandouras, I., and Daly, P. W.: Motion of flux transfer events: a test of the Cooling model, Ann. Geophys., 25, 1669-1690, doi:10.5194/angeo25-1669-2007, 2007.

Fear, R. C., Milan, S. E., Fazakerley, A. N., Lucek, E. A., Cowley, S. W. H., and Dandouras, I.: The azimuthal extent of three flux transfer events, Ann. Geophys., 26, 2353-2369, doi:10.5194/angeo-26-2353-2008, 2008.

Fear, R. C., Milan, S. E., Lucek, E. A., Cowley, S. W. H., and Fazakerley, A. N.: Mixed Azimuthal Scales of Flux Transfer Events, in: The Cluster Active Archive - Studying the Earth's Space Plasma Environment, edited by: Laakso, H., Taylor, M., and Escoubet, C. P., Astrophys. Space Sci. Proc., pp. 389398, Springer Netherlands, Dordrecht, doi:10.1007/978-90-4813499-1_27, 2010a.

Fear, R. C., Milan, S. E., Raeder, J., and Sibeck, D. G.: Asymmetry in the bipolar signatures of flux transfer events, J. Geophys. Res., 115, A11217, doi:10.1029/2010JA015363, 2010 b.

Fear, R. C., Palmroth, M., and Milan, S. E.: Seasonal and clock angle control of the location of flux transfer event signatures at the magnetopause, accepted, J. Geophys. Res., accepted, doi:10.1029/2011JA017235, 2012.

Gonzalez, W. D. and Mozer F. S.: A Quantitative Model for the Potential Resulting from Reconnection with an Arbitrary Interplanetary Magnetic Field, J. Geophys. Res., 79, 4186-4194, doi:10.1029/JA079i028p04186, 1974.

Honkonen, I., Palmroth, M., Pulkkinen, T. I., Janhunen, P., and Aikio, A.: On large plasmoid formation in a global magnetohydrodynamic simulation, Ann. Geophys., 29, 167-179, doi:10.5194/angeo-29-167-2011, 2011.

Janhunen, P.: GUMICS-3: A global ionosphere-magnetosphere coupling simulation with high ionospheric resolution, in: Proceedings of Environmental Modelling for Space-Based Applications, 18-20 September 1996, Eur. Space Agency Spec. Publ., ESA SP-392, 1996.

Kawano, H. and Russell, C. T.: Survey of flux transfer events observed with the ISEE 1 spacecraft: Rotational polarity 
and the source region, J. Geophys. Res., 101, 27299-27308, doi:10.1029/96JA02703, 1996.

Kawano, H. and Russell, C. T.: Survey of flux transfer events observed with the ISEE 1 spacecraft: Dependence on the interplanetary magnetic field, J. Geophys. Res., 102, 11307-11313, doi:10.1029/97JA00481, 1997.

Korotova, G. I., Sibeck, D. G., and Rosenberg, T.: Seasonal dependence of Interball flux transfer events, Geophys. Res. Lett., 35, L05106, doi:10.1029/2008GL033254, 2008.

Laitinen, T. V., Janhunen, P., Pulkkinen, T. I., Palmroth, M., and Koskinen, H. E. J.: On the characterization of magnetic reconnection in global MHD simulations, Ann. Geophys., 24, 30593069, doi:10.5194/angeo-24-3059-2006, 2006.

Lee, L. C. and Fu, Z. F.: A theory of magnetic flux transfer at the Earth's magnetopause, Geophys. Res. Lett., 12, 105-108, doi:10.1029/GL012i002p00105, 1985.

Lockwood, M. and Hapgood, M. A.: On the cause of a magnetospheric flux transfer event, J. Geophys. Res., 103, 26453-26478, doi:10.1029/98JA02244, 1998.

Lundin, R. and Evans, D. S.: Boundary layer plasmas as a source for high-latitude, early afternoon, auroral arcs, Planet. Space Sci., 33, 1389-1406, 1985.

Milan, S. E., Lester, M., Cowley, S. W. H., and Brittnacher, M.: Convection and auroral response to a southward turning of the IMF: Polar UVI, CUTLASS, and IMAGE signatures of transient magnetic flux transfer at the magnetopause, J. Geophys. Res., 105, 15741-15755, doi:10.1029/2000JA900022, 2000.

Palmroth, M., Pulkkinen, T. I., Janhunen, P., and Wu, C.-C.: Stormtime energy transfer in global MHD simulation, J. Geophys. Res., 108, 1048, doi:10.1029/2002JA009446, 2003.

Palmroth, M., Laitinen, T. V., and Pulkkinen, T. I.: Magnetopause energy and mass transfer: results from a global MHD simulation, Ann. Geophys., 24, 3467-3480, doi:10.5194/angeo-243467-2006, 2006.

Palmroth, M., Laitinen, T. V., Anekallu, C. R., Pulkkinen, T. I., Dunlop, M., Lucek, E. A., and Dandouras, I.: Spatial dependence of magnetopause energy transfer: Cluster measurements verifying global simulations, Ann. Geophys., 29, 823838, doi:10.5194/angeo-29-823-2011, 2011.

Papadopoulos, K., Goodrich, C., Wiltberger, M., Lopez, R., and Lyon, J. G.: The physics of substorms as revealed by the ISTP, Phys. Chem. Earth, 24, 189-202, 1999.

Raeder, J.: Flux Transfer Events: 1. generation mechanism for strong southward IMF, Ann. Geophys., 24, 381-392, doi:10.5194/angeo-24-381-2006, 2006.
Rijnbeek, R. P., Cowley, S. W. H., Southwood, D. J., and Russell, C. T.: Observations of reverse polarity flux transfer events at the Earth's dayside magnetopause, Nature, 300, 23-26, doi:10.1038/300023a0, 1982.

Rosenqvist, L., Buchert, S., Opgenoorth, H., Vaivads, A., and Lu, G.: Magnetospheric energy budget during huge geomagnetic activity using Cluster and ground-based data, J. Geophys. Res., 111, A10211, doi:10.1029/2006JA011608, 2006.

Rosenqvist, L., Vaivads, A., Retinò, A., Phan, T., Opgenoorth, H. J., Dandouras, I., and Buchert, S.: Modulated reconnection rate and energy conversion at the magnetopause under steady IMF conditions, Geophys. Res., Lett., 35, L08104, doi:10.1029/2007GL032868, 2008.

Russell, C. T. and Elphic, R. C.: Initial ISEE magnetometer results: magnetopause observations, Space Sci. Rev., 22, 681-715, doi:10.1007/BF00212619, 1978.

Russell, C. T. and Elphic, R. C.: ISEE observations of flux transfer events at the dayside magnetopause, Geophys. Res. Lett., 6, 33 36, doi:10.1029/GL006i001p00033, 1979.

Russell, C. T. and McPherron, R. L.: Semiannual variation of geomagnetic activity, J. Geophys. Res., 78, 92-108, 1973.

Russell, C. T., Luhmann, J. G., and Berchem, J.: On the source region of flux transfer events, Adv. Space Res., 5, 363-368, doi:10.1016/0273-1177(85)90162-0, 1985.

Saunders, M. A., Russell, C. T., and Sckopke, N.: Flux transfer events: Scale size and interior structure, Geophys. Res. Lett., 11 , 131-134, doi:10.1029/GL011i002p00131, 1984.

Scholer, M.: Magnetic flux transfer at the magnetopause based on single X line bursty reconnection, Geophys. Res. Lett., 15, 291294, doi:10.1029/GL015i004p00291, 1988.

Shukhtina, M. A., Gordeev, E. I., and Sergeev, V. A.: Timevarying magnetotail magnetic flux calculation: a test of the method, Ann. Geophys., 27, 1583-1591, doi:10.5194/angeo-271583-2009, 2009.

Siscoe, G. L. and Cummings, W. D.: On the cause of geomagnetic bays, Planet. Space Sci., 17, 1795-1802, 1969.

Sonnerup, B. U. O.: Experimental tests of FTE theories, Adv. Space Res., 8, 263-272, doi:10.1016/0273-1177(88)90140-8, 1988.

Southwood, D. J., Farrugia, C. J., and Saunders, M. A.: What are flux transfer events?, Planet. Space Sci., 36, 503-508, doi:10.1016/0032-0633(88)90109-2, 1988. 